\title{
Evaluación de riesgo para la salud pública: nuevo instrumento de gestión ambiental para Chile
}

\author{
PATRICIA MATUS C. ${ }^{1, a}$
}

\section{Evaluation of public health risk. A new instru- ment for environmental management in Chile}

One of the main challenges in environmental management is to prevent the public health impact of projects that can cause pollution. To tackle this problem, the new Chilean bill on environmental management has defined the need to determine the potential health risks of a given Project. This paper gives a summary of the method used for risk evaluation and its evolution. Its incorporation in the Environmental Impact Evaluation System is proposed, to guarantee an effective prevention of the potential risks on health of new projects.

(Rev Med Chile 2011; 139: 1075-1080).

Key words: Air pollution; Environment; Public health.

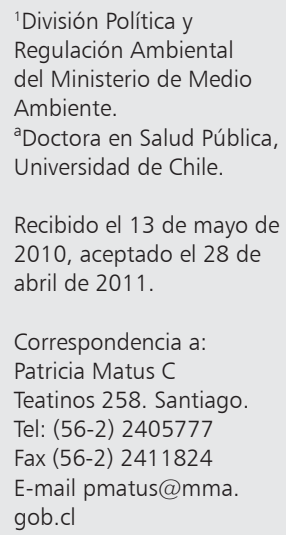

L a ley sobre Bases Generales del Medio establecida en Chile en el año 1995 recientemente fue modificada, incorporándose a la gestión ambiental nuevas instituciones, procedimientos e instrumentos de gestión ambiental ${ }^{1}$. Los cambios más trascendentales están relacionados con la creación de una Superintendencia de Fiscalización Ambiental que tendrá por finalidad fiscalizar el cumplimiento de todos los instrumentos de carácter ambiental. A la par se creará un tribunal ambiental para la resolución de controversias que se puedan dar en el ejercicio de las normas y exigencias ambientales definidas por el Ministerio del Medio Ambiente.

Los instrumentos de gestión ambiental que estarán bajo el control del nuevo organismo son las Resoluciones de Calificación Ambiental, las Normas Primarias o Secundarias de Calidad Ambiental, las Normas de Emisión, los Planes de Prevención de la Contaminación y de Descontaminación y los Planes de Manejo de recursos naturales.

Un cambio importante que puede tener trascendencia para la salud ambiental, pues asegura la evaluación sistemática del impacto de la contaminación potencial sobre las personas, es la introducción de la evaluación de riesgo en la gestión ambiental. Específicamente se incorpora la evaluación de riesgo de productos químicos, organismos genéticamente modificados y otras sustancias que puedan afectar el medio ambiente y la evaluación de riesgo para la salud de las personas en el procedimiento de Evaluación del Impacto Ambiental.

El Sistema de Evaluación de Impacto Ambiental es un instrumento eminentemente preventivo, es decir, se ejecuta sobre la base de ideas de proyectos y no sobre instalaciones que estén operando. En la etapa de diseño, los proyectos son analizados para evaluar el grado de impacto ambiental que ellos potencialmente pueden producir sobre el medio ambiente. En dicho proceso se mide la línea base actual del entorno ambiental y se estiman los posibles cambios potencialmente provocados por las emisiones, procesos y actividades que el proyecto producirá una vez que entre en fase de desarrollo y operación.

El parlamento ha planteado que "Cuando el Proyecto deba presentar un Estudio de Impacto Ambiental por generar alguno de los efectos, características o circunstancias señaladas en la letra a) del artículo 11, y no existiera Norma Primaria o de 
Emisión en Chile o en los Estados de referencia que señale el Reglamento, el proponente deberá considerar un capitulo específico relativo a los potenciales riesgos que el proyecto podría generar en la salud de las personas".

Establece por lo tanto, el nuevo texto legal, que para los proyectos que por su naturaleza generen riesgo para la salud de la población debido a la cantidad y calidad de sus efluentes, emisiones o residuos (literal a del artículo 11 de la Ley 19.300) el Estudio de Impacto Ambiental deberá incluir un capítulo sobre sus riesgos potenciales a las personas. Esto no sería necesario de existir normas primarias de calidad o de emisión vigentes, que puedan ser contrastadas con las emisiones potenciales. En este caso solamente se requeriría evaluar el cumplimiento de tales exigencias.

La variedad de elementos químicos, físicos y biológicos que pueden ser liberados por las actividades antropogénicas resulta ser tan amplia que con bastante probabilidad una gama importante de los proyectos que requieran un estudio de impacto ambiental para poder ser ejecutados, requerirán también la elaboración del mencionado capítulo, pues muchas de las emisiones de dichos proyectos no se encuentran reguladas.

Existen otros impactos ambientales, como son el uso del recurso hídrico, o algunos servicios ambientales y sus efectos en las poblaciones humanas que pueden ser evaluados dentro del marco de los impactos eco- sistémicos (letra b del artículo 11) y no en su relación con la salud de las personas.

Interesa por lo tanto, avanzar en los conceptos de evaluación de riesgo, ya que la ley no introdujo definiciones, ni estableció criterios ni procedimientos específicos para llevar a cabo este tipo de análisis.

Esta revisión tiene por finalidad identificar las alternativas de evaluación del impacto sobre la salud o de evaluación de riesgo que pudieran ser adoptadas para cumplir con los objetivos planteados por el legislador chileno y propone una modalidad que considera su articulación con la evaluación ambiental actualmente imperante en el país.

\section{Sobre la evaluación de riesgo ambiental}

A mediados del siglo pasado y producto del creciente interés de las sociedades desarrolladas de definir metodologías para la toma de decisión ambiental, se estableció la metodología de evaluación de riesgo $\mathrm{o}^{2-5}$.
Dicha metodología contempla los siguientes pasos:

1. Identificación del peligro (fuente del daño potencial).

2. Caracterización de la exposición (tipo, vías de exposición).

3. Evaluación de la exposición (extensión espacial y temporal de la exposición).

4. Caracterización del riesgo (relación entre exposición y efectos).

5. Gestión del riesgo (elección de acciones para eliminar o reducir el peligro, incluye la medición sistemática de la exposición y cambios a las alternativas cuando se cuente con nueva información).

6. Comunicación del peligro y el riesgo a los posibles afectados.

A modo de ejemplo en un proyecto de reciclaje de baterías se identifica la intoxicación plúmbica aguda o crónica como uno de los peligros potenciales. Se deben analizar todas las vías probables de exposición, es decir, todos los medios (aire-aguasuelo-alimentos) que pudieran permitir el contacto entre el plomo y los seres humanos. Estimar a partir de las emisiones teóricas del proyecto la magnitud de la exposición (concentraciones en los distintos medios) y la frecuencia de exposición. Posteriormente, se establece la relación dosis-respuesta, es decir, estimar sobre la base de la cantidad de exposición, la cantidad de daño potencial esperado. En virtud de dichos resultados se establecen los límites aceptables de emisión (normas de emisión) para proteger a la comunidad y/o las medidas de protección específica para los trabajadores (rangos de exposición biológica aceptables). La gestión del riesgo comprende también una adecuada información hacia todos los involucrados, en este caso población general, trabajadores, empresa y profesionales que deben controlar la situación de la salud ambiental de la localidad en donde el proyecto se instalará.

La evaluación de riesgo se facilita bastante cuando el problema a estudiar tiene una realidad física comprobable (sitio contaminado o fuente de contaminación activa) y no es solamente un proyecto o idea, como es el caso de los Estudios de Impacto Ambiental, pues las estimaciones se pueden hacer a partir de mediciones concretas en el sitio del problema y no a partir de presunciones de emisiones como ocurre en el caso de la evaluación de proyectos. 
La evaluación de riesgo se ha utilizado principalmente en la definición de límites de emisión o el establecimiento de estándares de calidad. En la actualidad uno de los últimos desarrollos de ésta metodología es la evaluación de riesgo de sustancias peligrosas en la Unión Europea, regulación $\mathrm{REACH}$, para otorgar permisos de comercialización de sustancias químicas en dicho continente ${ }^{6}$.

Se ha argumentado que la evaluación de riesgo, siendo un proceso lineal y específico para sustancias químicas, no permite enfrentar adecuadamente la característica de complejidad que tienen los riesgos ambientales, que suelen ser las mezclas de sustancias químicas y conllevan efectos subjetivos en la población.

Es así como en los años recientes se han establecido desarrollos para la evaluación incorporando distintos agentes así como variadas disciplinas a la evaluación. La nominación evaluación integrada de riesgo, da cuenta de esta nueva modalidad. Se entiende por "el enfoque científico que combina el proceso de estimación del riesgo en humanos, la biota y los recursos naturales en un mismo proceso"7. También se ha incorporado a la evaluación la participación de actores concernidos -stakeholder- y la consideración de los efectos socio-económicos y culturales en la evaluación y selección de alternativas de solución.

En paralelo se ha desarrollado el concepto de Evaluación Comparativa de Riesgo, desarrollada como una extensión al ámbito ambiental de la evaluación de carga de enfermedad. Murray y cols definen a ésta metodología como "evaluación sistemática de los cambios en la salud de la población que pueden resultar de los cambios en la distribución de la exposición a un factor de riesgo o a un grupos de factores de riesgo". Esta metodología fue usada en Chile para el cálculo de la carga atribuible a factores de riesgo del estudio de Carga de Enfermedad realizado a petición del Ministerio de Salud por la Universidad Católica en el año $2007^{8}$, y permitió identificar a la presión arterial elevada, al consumo de sal y alcohol y a la contaminación atmosférica como los factores de riesgo con mayor peso relativo en la carga de enfermedad total del país.

Más recientemente Briggs (2008) ha propuesto la evaluación del impacto en salud ambiental integrada como un medio para valorar los problemas sobre la salud de la población derivados de las intervenciones sobre el medio ambiente, de una forma que tome en cuenta la complejidad, las interdependencias e incertidumbres del mundo real ${ }^{9}$.

Dicho método se diferencia del tradicional en que presta mayor atención a una etapa previa a la evaluación propiamente tal, denominada Issueframming, que tiene por finalidad asegurar que el objetivo y alcance de la evaluación se haya definido adecuadamente, así como la selección de escenarios y sus indicadores. Luego viene la etapa de diseño, en la que el modelo conceptual elaborado en la primera etapa se transforma en un protocolo detallado para la evaluación. Posteriormente, viene la etapa de ejecución de la evaluación que comprende las etapas de la evaluación de riesgo tradicional (identificación del peligro, evaluación de la exposición y caracterización del riesgo). Por último, la etapa de valoración en la que los resultados se sintetizan y se interpretan. comprende la evaluación y ponderación de los resultados para los distintos escenarios y el ordenamiento de las opciones de política en términos de su aceptabilidad y efectividad. Esta modalidad de evaluación se ajusta a lo que la institucionalidad chilena ha denominado evaluación ambiental estratégica de políticas, planes y programas.

\section{El sistema de evaluación de impacto ambiental en Chile}

La evaluación ambiental de proyectos de inversión es una de las principales herramientas de gestión ambiental establecida en Chile desde el año 1995. Consiste en un proceso que tiene por finalidad que las inversiones productivas se evalúen en forma preventiva, es decir, en etapa de diseño los potenciales impactos sobre el medio ambiente, para proponer medidas de mitigación y/o reparación ambiental cuando así lo ameriten. El sistema chileno contempla además el otorgamiento de una serie de permisos ambientales, estableciéndose una ventanilla única por parte de los organismos de gobierno responsables del control y vigilancia de las actividades productivas, y la participación ciudadana, por medio de un procedimiento a cargo de los proponentes de los proyectos y administrado por el Servicio de Evaluación Ambiental ${ }^{10}$.

La evaluación del impacto sigue un procedimiento, con plazos y formalidades que, de manera resumida, consiste en la caracterización de la línea de base ambiental del lugar en donde se emplazará el proyecto, la descripción del proyecto, la estima- 
ción de impactos ambientales y el establecimiento de medidas de control de dichos impactos.

El Estudio de Impacto Ambiental, más específicamente, debe contener la descripción del proyecto o actividad; la descripción de la línea base; una descripción de los efectos que dan origen a la necesidad de efectuar un estudio de impacto ambiental; la identificación, predicción y evaluación de los impactos ambientales del proyecto o actividad, incluidas las situaciones de riesgo; un plan de medidas de mitigación, reparación y compensación; las medidas de prevención de riesgos y control de accidentes; $y$ un plan de seguimiento de las variables ambientales que dieron origen al estudio ${ }^{11}$.

La ciudadanía puede participar por medio de eventos públicos en donde los proponentes exponen el proyecto y responde las dudas que pueda suscitar el proyecto, sus emisiones o intervención en el entorno. Para facilitar dicha participación el proponente debe publicar un resumen del estudio de impacto ambiental en un diario de circulación nacional y en el Diario Oficial. Las organizaciones ciudadanas con personalidad jurídica y los individuos naturales directamente afectados por el proyecto pueden conocer el estudio in extenso, formular observaciones por escrito, recibir respuesta por parte de las autoridades respecto de ellas y reclamar ante el director nacional de la CONAMA, si consideran que sus opiniones no fueron debidamente ponderadas en el momento de la evaluación del proyecto.

Tradicionalmente se ha evaluado el componente de salud dentro de la línea de base social de los estudios de evaluación de impacto ambiental, se han estimado las emisiones y contrastado éstas con el cumplimiento de normas primarias de calidad ambiental (principalmente de aire) y/ o de emisión, por lo que los efectos potenciales sobre la salud de los proyectos quedaban circunscritos al cumplimiento de la normativa ambiental, apareciendo muchas veces a nivel de la participación ciudadana preguntas sobre daños potenciales sobre la salud, no suficientemente analizados en los respectivos estudios.

Cómo incorporar el análisis de riesgo sobre la salud en el sistema de evaluación del impacto ambiental

El sistema chileno de evaluación ambiental está concebido para identificar formas de optimizar los impactos ambientales positivos y minimizar, atenuar o contrarrestar los impactos ambientales negativos ${ }^{12}$, considerando como impacto ambiental a la alteración del medio ambiente provocada, directa o indirectamente, por un proyecto o actividad en un área determinada, $y$, al medio ambiente, como el sistema global constituido por elementos naturales y artificiales de naturaleza física, química o biológica, socioculturales y sus interacciones, en permanente modificación por la acción humana o natural y que rige y condiciona la existencia y el desarrollo de la vida en sus múltiples manifestaciones $^{13}$. Este procedimiento tiene por limitación formal el que no extiende su análisis al pronóstico de los daños y beneficios potenciales sobre la salud que la actividad o proyecto puede provocar sobre los trabajadores, dado que en la institucionalidad chilena, el entorno del trabajo se encuentra regulado por leyes y reglamentos específicos dependientes del Código Sanitario (ley 17.748 de protección frente a accidentes y enfermedades profesionales y reglamento 594 sobre condiciones ambientales mínimas de lugares de trabajo).

Siguiendo las indicaciones generales establecidas para la evaluación ambiental, el análisis de riesgo debiera contemplar los pasos señalados en la Figura 1, usando los métodos señalados en la Figura 2. Interesa pronosticar los daños y beneficios potenciales sobre la salud que la actividad o proyecto puede provocar sobre los trabajadores y la comunidad aledaña al lugar en donde la instalación o actividad se desarrollará.

El pronóstico o predicción de los posibles impactos sobre la salud en una población a causa de un proyecto o actividad se establece a partir de una secuencia analítica que comprende el análisis del proyecto, con la estimación de la contaminación futura y el análisis de la localidad que potencialmente será impactada. El análisis de la localidad comprende tanto aspectos físicos y demográficos como de situación de salud.

La predicción del impacto sobre la salud se realiza mediante la integración del conocimiento sobre la cantidad de contaminantes presentes en todos los medios de exposición (agua, aire, suelo, flora y fauna), el tipo de exposición potencial y el análisis de la población receptora (tamaño, edad, género, estado de salud, y hábitos) ${ }^{14}$.

Para la elaboración de la línea base en salud corresponde realizar un diagnóstico de situación de salud de la población que habita en el área de 

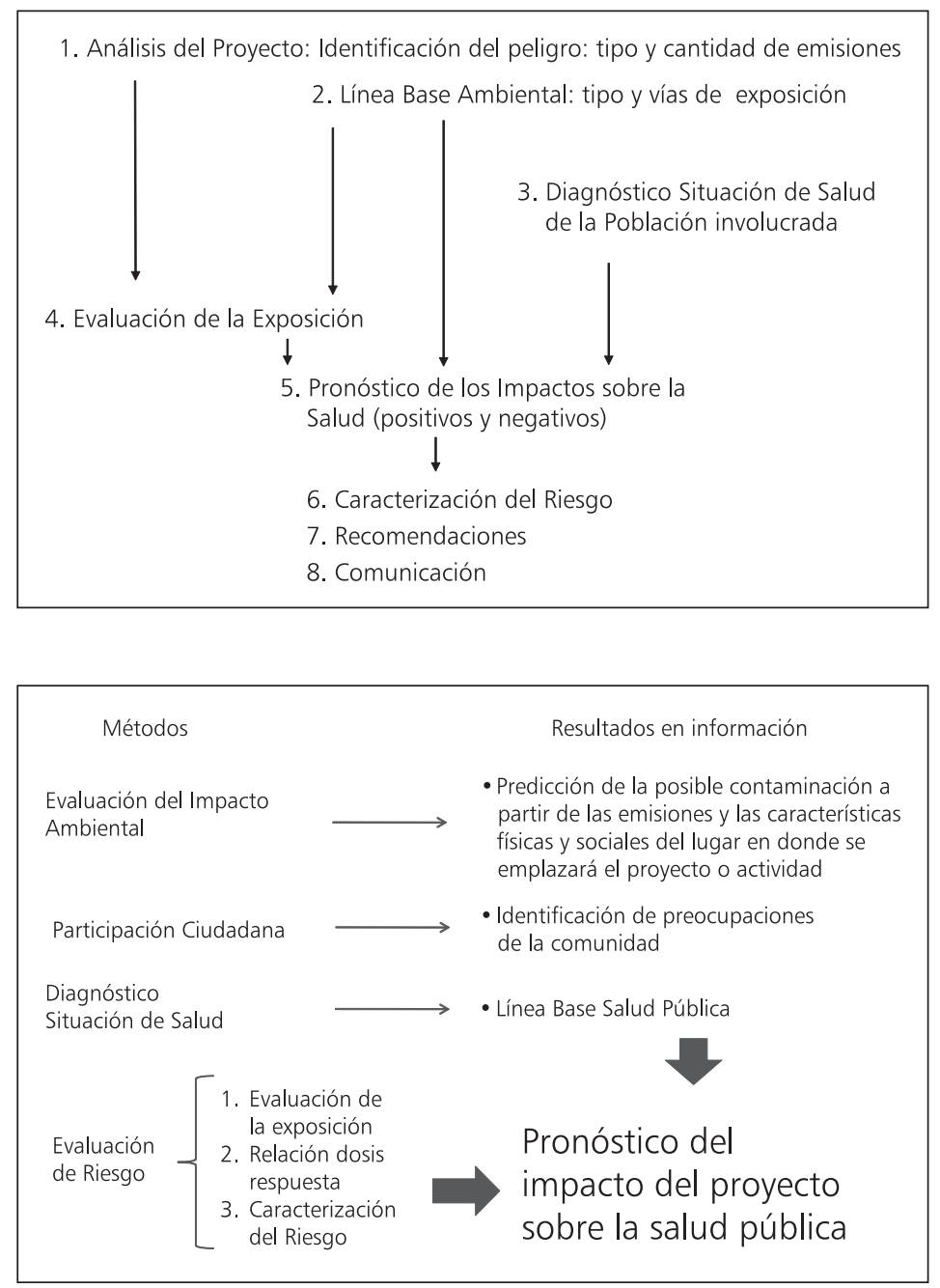

Figura 1. Secuencia para evaluar el impacto sobre la salud de un proyecto.
Figura 2. Métodos y requerimientos de información para efectuar un análisis de riesgo del impacto de un proyecto o actividad en un Estudio de Impacto Ambiental. impacto del proyecto. Es decir, a la descripción de las características socio-demográficas de la población, con particular acento sobre su estructura poblacional, pues poblaciones envejecidas pueden ser más vulnerables a ciertas noxas ambientales que las poblaciones jóvenes. Las características de la mortalidad y morbilidad, idealmente comparadas con promedios nacionales $\mathrm{y} / \mathrm{o}$ regionales para identificar los principales daños a la salud presentes en la población y un resumen de los recursos de atención de salud de que la población dispone. Las características físicas de la región (calidad del agua, capacidad de dispersión de la cuenca atmosférica, etc) son importantes pues permitirán, sobre la base de las emisiones proyectadas de la actividad, predecir el impacto ambiental sobre la calidad del agua, el aire y el manejo de residuos sólidos. Estos aspectos son normalmente evaluados durante el proceso de elaboración del estudio de impacto ambiental. Esa misma información permitirá conocer valores potenciales de contaminantes en los medios de exposición de la población. Su comparación con estándares de exposición y/o normas, en el caso de tener umbral, y con niveles aceptables de riesgo, cuando los contaminantes tengan efectos sin nivel de umbral, permitirán realizar la caracterización del riesgo. La estimación del impacto de dichas emisiones sobre la salud pública requiere de un procedimiento de análisis y apreciación del riesgo sobre la población expuesta. Los efectos potenciales serán distintos en magnitud y tipo dependiendo del tamaño y 
características de la población expuesta. A modo de ejemplo, en población activa laboral no se evidencian los mismos efectos que sobre la población general. Esto se denomina efecto trabajador sano.

La evaluación del riesgo debiera contemplar dar respuesta a las inquietudes sobre salud de la población, por esto dicha evaluación debiera conocer cuáles son las preguntas frecuentes que la población hace al momento de la participación ciudadana. El tipo de efecto a estudiar (agudo, crónico, alteración del desarrollo, efecto carcinogénico, etc) puede establecerse a partir de la revisión bibliográfica de los efectos de proyectos similares y/o sus emisiones. Dicha revisión permitirá identificar los problemas potenciales a la salud esperables de ejecutarse el proyecto. Como los daños a la salud son multi-causales, interesa, para realizar evaluaciones ulteriores, conocer la situación de base de dichas patologías en el área de impacto del proyecto (línea base). Los estudios de evaluación de impacto ambiental estipulan la elaboración de un plan de seguimiento de las variables ambientales que dieron origen al estudio. El seguimiento de dichas variables, bastará para evaluar las medidas de mitigación pues generalmente se traducen en normas de emisión o de calidad que fijan niveles de riesgo aceptables para la población. Frente a grandes incertidumbres podría ser interesante incorporar el seguimiento de los potenciales efectos adversos más severos ${ }^{15}$.

En conclusión, la evaluación sistemática del impacto de los proyectos sobre la salud de las personas expuestas, por medio de la adopción de la evaluación de riesgo, mejorará el procedimiento de evaluación de impacto ambiental permitiendo prevenir daños sobre las personas a causa de la contaminación.

\section{Referencias}

1. CONAMA Ley 20417 del 12 de enero 2010. www.conama.cl[Consultado el 15 de marzo de 2010].

2. Ricci P. Environmental and Health Risk Assessment and Management. Principles and Practices. Springer, 2006.
3. Asante K. Principals elements of public health risk assessment for chemical exposure problems. En: AllowayJ, Trevors J Editores London, UK: Editoral Kluwer Academic Publishers; 2002, 71-6.

4. World Bank. Environmental Assessment Sourcebook. Vols I-III. Environmental department. Washington DC: World Bank Technical Paper 1991, № 139, 140 y 154.

5. U.S Protection Agency (EPA) Risk Assessment guidance for superfund. Voll Human Health Evaluation Manual (Part A). EPA/540:1-891002, Washington DC: EPA, 1989.

6. Parlamento Europeo Reglamento (CE) no 1907/2006 relativo al registro, la evaluación, la autorización y la restricción de las sustancias y preparados químicos (REACH). Euro-Lex -32006R1907. Disponible en http:// eur-lex.europa.eu [Consultado el 3 de mayo de 2010].

7. WHO/IPCS Integrated Risk Assessment. Report prepared for the WHO/UNEP/ILO International Programme on Chemical Safety. 2001. Disponible http://www.who. int/ipcs/publications [Consultado el 16 de abril de 2010].

8. Ministerio de Salud. Informe final estudio de carga de enfermedad y carga atribuible, Julio 2008. Disponible en www.minsal.cl [Consultado el 10 de abril de 2010].

9. Briggs D. A framework for integrated environmental health impact assessment of systemic risks Environmental Health 2008, 7: 61.

10. CONAMA. DS n 95/2001 Reglamento de Evaluación de Impacto Ambiental. Disponible en www.conama.cl. [Consultado el 16 de marzo de 2010].

11. Astorga E. Sistema de Evaluación de Impacto Ambiental, régimen Jurídico en especial aplicado a la actividad minera; Ed. Conosur Ltad., Santiago, 2000.

12. Espinoza G. Gestión y Fundamentos de Evaluación de Impacto Ambiental. Banco Interamericano de Desarrollo, 2006.

13. CONAMA. Ley sobre bases generales del medio ambiente. Disponible en www.conama.cl. [Consultado el 16 de abril de 2010].

14. Fehr R. Environmental Health Impact Assessment. Evaluation of a ten-step model. Epidemiology 1999,5:618625.

15. García M, Prieto V, Molina E, Junco R, Barceló C, Diego F. Evaluación del impacto en salud de proyectos de inversión. Experiencia cubana. Rev. Cubana Hig Epidemiol $2005 ; 43$ (3). 